\title{
A Mobile-Agent-Based Adaptive Data Fusion Algorithm for Multiple Signal Ensembles in Wireless Sensor Networks
}

\author{
Tianjing WANG ${ }^{1}$, Zhen YANG ${ }^{2}$, Guoqing LIU ${ }^{1}$ \\ ${ }^{1}$ College of Science, Nanjing University of Technology, Nanjing, China \\ ${ }^{2}$ Institute of Signal and Information Processing, Nanjing University of Posts\&Telecommunications, Nanjing, China \\ Email: tjingwang@126.com \\ Received June 8, 2009; revised August 17, 2009; accepted August 18, 2009
}

\begin{abstract}
Distributed Compressed Sensing (DCS) is an emerging field that exploits both intra- and inter-signal correlation structures and enables new distributed coding algorithms for multiple signal ensembles in wireless sensor networks. The DCS theory rests on the joint sparsity of a multi-signal ensemble. In this paper we propose a new mobile-agent-based Adaptive Data Fusion (ADF) algorithm to determine the minimum number of measurements each node required for perfectly joint reconstruction of multiple signal ensembles. We theoretically show that $\mathrm{ADF}$ provides the optimal strategy with as minimum total number of measurements as possible and hence reduces communication cost and network load. Simulation results indicate that ADF enjoys better performance than DCS and mobile-agent-based full data fusion algorithm including reconstruction performance and network energy efficiency.
\end{abstract}

Keywords: Wireless Sensor Networks, Mobile Agent, Compressed Sensing, Distributed Compressed Sensing, Joint Sparsity, Joint Reconstruction

\section{Introduction}

Wireless Sensor Networks (WSN) are an emerging technology that promises an ability to monitor the physical world via spatially distributed networks of small and inexpensive wireless sensor nodes that have the ability to self-organize into a well-connected network. The communication tasks consume the limited power available at such sensor nodes and, therefore, in order to ensure their sustained operations, the power consumption must be kept to minimum. Different from transmission cost, the computational cost may be negligible for some applications. For example, WSN monitoring field temperature may use simple functions which essentially are of insignificant cost. Consequently, a major challenge in the design of WSN is developing schemes that extract relevant information about the sensor data with the minimum energy consumption, especially with transmission cost. In order to reduce transmission of sensor data, a new framework for single signal sensing and compression has developed recently under the rubric of Com-

\footnotetext{
*Supported by the Young Teacher Academic Fund of Nanjing Univer-
} sity of Technology pressed Sensing (CS) [1-3]. The implications of CS are promising for many applications, especially sensing signals that have a sparse representation in some basis [4-6]. Based on the intra-signal correlations (between samples in each signal), CS can perfectly reconstruct a compressible signal from remarkably few linear measurements. In WSN, however, a large number of sensor nodes presumably observe related phenomena and are programmed to perform a variety of signals acquisition tasks. These signals have high correlations and need to be jointly processed, so the independently encoding and decoding theory and practice of single signal in a CS framework can not satisfy such applications. Fortunately, the ensemble of signals that sensor nodes acquired can be expected to possess some joint structure, or inter-signal correlations (between samples across signals), in addition to the intra-signal correlation of single signal. Most existing coding algorithms [7,8], however, exploit only inter-signal correlations and not intra-signal correlations. A new Distributed Compressed Sensing (DCS) theory exploits both intra-signal and inter-signal correlation structures of multiple signal ensembles [9-11]. In a typical DCS scenario, each individual node independently encodes its signal by CS framework and then transmits 
the measurements to a distant Sink node (Sink) by multiple skips. Under the right conditions, Sink can jointly reconstruct multiple signal ensembles precisely. However, we note that the network traffic may be extremely heavy in DCS, resulting in poor performance of the system, when the number of sensor nodes is big and the amount of sensing signals is large. Furthermore, nodes can not know the suited minimum number of measurements that they need to transmit to Sink under DCS framework. In order to guarantee perfect reconstruction, each node has to transmit enough measurements. This means that DCS only utilizes the inter-signal correlations in jointly decoding processes, but not in jointly encoding processes.

In this paper, we design a mobile-agent-based Adaptive Data Fusion (ADF) algorithm for multiple signal ensembles which is inspired by DCS. Instead of passing large amounts of independently encoding measurements by DCS in each individual node over the network, Mobile Agent (MA) can fuse multiple signals from node to node along a shortest path, based on Global Closest First (GCF) heuristics algorithm [12]. According to the sparse property of single signal and the joint sparsity of multiple signal ensembles under the results of data fusion, each node can determine the minimum number of measurements needed to transmit to Sink and effectively reduces the transmission cost. Extensive experiments in session 4 demonstrate that the energy efficiency and the reconstruction performance of ADF are more excellent than DSC and mobile-agent-based Full Data Fusion (FDF) algorithm.

The organization of this paper is as follows. Section 2 overviews a joint sparsity model in DCS. Single signal and multiple signals reconstruction methods are discussed respectively. Section 3 introduces our two mobile-agent-based data fusion algorithms: Full Data Fusion (FDF) and Adaptive Data Fusion (ADF). Detailed theoretical analyses indicate that FDF and ADF are more energy efficient than DCS, sufficiently utilizing intrasignal and inter-signal correlation of multiple signal ensembles. Experiments in Section 4 confirm that ADF indeed reduces large amounts of total transmission cost and the number of total measurements compared to DCS and FDF. Section 5 describes conclusions.

\section{Distributed Compressed Sensing}

\subsection{Joint Sparsity Model}

The recently introduced theory of DCS enables a new distributed coding algorithm that exploits both intra- and inter-signal correlation structures of multiple signals [9]. In this paper, we focus on a Joint Sparsity Models (JSM, sparse common component +innovations), which can be improved by mobile-agent-based data fusion. For exam- ple, a practical situation well-modeled by the JSM is a group of sensors $\left\{S_{l}, \ldots S_{J}\right\}$ measuring temperatures at a number of outdoor locations throughout the day. The temperature readings $x_{l}(l \in\{1,2, \ldots J\})$ have both temporal (intra-signal) and spatial (inter-signal) correlations. Global factors, such as the sun and prevailing winds, could have an effect that is both common to all sensors and structured enough to permit sparse representation in some basis. More local factors, such as shade, water, or animals, could contribute localized innovations that are also structured (and hence sparse in some basis). A similar scenario could be imagined for a network of sensors recording light intensities, air pressure, or other phenomena. All of these scenarios correspond to measuring properties of physical processes that change smoothly in time and in space and thus are highly correlated.

We adopt language and notation from [9]. Assume that there exists a known basis $\Psi=\left\{\psi_{i} \mid \psi_{i} \in R^{m}, i=1, \mathrm{~L}, m\right\}$ in which a signal $x_{l}=\left\{x_{l}(1), \mathrm{L}, x_{l}(m)\right\}^{T} \in R^{m}(l \in\{1,2, \mathrm{~L}, J\})$ can be sparsely represented as $x_{l}=\Psi \theta_{l}$, where $\theta_{l}=\left(\theta_{l}(1), \mathrm{L}, \theta_{l}(m)\right)^{T}$ is a sparse coefficient vector and $\left\|\theta_{l}\right\|_{0}=k$. Thus, a compressible multiple signal ensemble $x_{1}, \mathrm{~L}, x_{J}$ shares a common sparse component while each single signal contains an innovation sparse component. That is

$$
\theta_{l}=\theta_{C}+\hat{\theta_{l}}, \quad l \in\{1,2, \mathrm{~L}, J\}
$$

with

$$
x_{C}=\Psi \theta_{C},\left\|\theta_{C}\right\|_{0}=k_{C}
$$

and

$$
\hat{x}_{l}=\Psi \hat{\theta_{l}},\left\|\hat{\theta_{l}}\right\|_{0}=k_{l} \quad\left(k_{l}<k_{C}\right)
$$

where $k_{c}$ is a common sparse parameter of $\theta_{c}$ and $k_{l}$ is an innovation sparse parameter of $\theta_{l}$.

Thus, the signal $x_{c}$ is common to all of $x_{l}(l \in\{1,2, \mathrm{~L}, J\})$ and has sparse coefficient vector $\theta_{c}$ in basis $\psi$, and the signal $\hat{x}_{l}(l \in\{1,2, \mathrm{~L}, J\})$ is the unique portions of $x_{l}$ and has sparse coefficient vector $\hat{\theta_{l}}$ in the same basis. Denote that $\Omega_{c}$ is a tight support set of the nonzero values in $\theta_{c}$ and $\Omega_{l}$ is a tight support set of the nonzero values in $\hat{\theta_{l}}$.

To make linear measurements, denote the measurement matrix $\Phi_{l}=\left(\varphi_{i j}\right)_{n_{l} \times m} \quad(l \in\{1,2, \mathrm{~L}, J\})$ for the multisignal ensembles, where a second basis matrix $\Phi_{l}$ is incoherent with $\Psi$. Thus, a small number of noiseless measurements $y_{l}=\Phi_{l} x_{l}=\left(y_{l}(1), \quad \mathrm{L}, y_{l}\left(n_{l}\right)\right)^{T}(l \in\{1,2, \mathrm{~L}, J\})$ contain sufficient information for approximate reconstruction [1,2]. Mathematically, this can be reduced to a standard linear algebra problem: we wish to find 
$x_{l}(l \in\{1,2, \mathrm{~L}, J\})$ which explains the measurements $y_{l}=\Phi_{l} x_{l}=\Phi_{l} \Psi \theta_{l} \quad(l \in\{1,2, \mathrm{~L}, J\})$.

\subsection{Joint Reconstruction of Multiple Signal Ensembles via $l_{1}$ Optimization}

To give ourselves a firm footing for analyses, we firstly consider single signal reconstruction based on the CS theory, mainly to exploit intra-signal structure at a single node. CS encodes a workable approximation of the single compressible signal $x_{l}(l \in\{1,2, \mathrm{~L}, J\})$ with $n_{l}=m$ sampling resources and signal reconstruction can be achieved by Basis Pursuit (BP) [1,2] or Orthogonal Matching Pursuit (OMP) [3]. OMP allows faster reconstruction at the expense of more measurements.

Formally, BP needs to solve the following $l_{1}$ optimization problem

$\left(\mathrm{P}_{1}\right) \min \left\|\theta_{l}\right\|_{1}$ subject to $y_{l}=\Phi_{l} x_{l}=\Phi_{l} \Psi \theta_{l}(l \in\{1,2, \mathrm{~L}, J\})(3)$

The simulation results in [2] state that there exists measurements $\mathrm{n} \geq \mathrm{ck}$ (oversampling factor $c \geq 4$ ) are required to reconstruct $x_{l}$ with high probability, using linear programming methods e.g. interior point method and simplex method. That is, an optimal reconstructed signal $x_{l}^{*}=\Psi \theta_{l}^{*}$ can be achieved by an optimal sparse solution $\theta_{l}^{*}$ of the problem $\left(\mathrm{P}_{1}\right)$.

In addition to single signal encoding and decoding methods, the jointly encoding and decoding methods of multiple signal ensembles are considered in DCS. DCS expects that 1) $c\left(k_{C}+k_{l}\right)(l \in\{1,2, \mathrm{~L}, J\})$ measurements suffice to reconstruct $\left.x_{1}, 2\right) c\left(k_{C}+\sum_{l=1}^{J} k_{l}\right)$ measurements suffice to reconstruct multiple signal ensembles $x_{1}, \ldots, x_{J}$, for there exists $k_{C}+\sum_{l=1}^{J} k_{l}$ nonzero elements in $x_{1}, \ldots, x_{J}$. Furthermore, the recovery problem can be formulated using matrices and vectors as

$$
\begin{gathered}
\theta=\left[\begin{array}{c}
\theta_{C} \\
\theta_{1} \\
\mathrm{M} \\
\theta_{J}
\end{array}\right], x=\left[\begin{array}{c}
x_{1} \\
\mathrm{M} \\
x_{J}
\end{array}\right], y=\left[\begin{array}{c}
y_{1} \\
\mathrm{M} \\
y_{J}
\end{array}\right], \\
\Phi 0=\left[\begin{array}{lll}
\Phi_{1} & \mathrm{~L} & 0 \\
& \mathrm{O} & \\
0 & \mathrm{~L} & \Phi_{J}
\end{array}\right], \quad \%=\left[\begin{array}{cccc}
\Psi & \Psi & \mathrm{L} & 0 \\
\mathrm{M} & & \mathrm{O} & \\
\Psi & 0 & & \Psi
\end{array}\right](4)
\end{gathered}
$$

In order to sufficiently utilize inter-signal correlation of multiple signal ensembles, we assume that $\Phi_{1}=\mathrm{L}=\Phi_{J}=\Phi$ and then $\$ \circ$ can be rewritten as $\$ \circ=\operatorname{diag}(\Phi, \mathrm{L}, \Phi)$. It is possible to let Sink previously send the same random seed to all sensor nodes in the interesting field and then the same pseudorandom matrix $\Phi$ can be generated using simple algorithm with seed at each node.

With sufficient sampling, DCS can reconstruct multiple signal ensembles by solving the following $l_{1}$ optimization problem

$$
\left(\mathrm{P}_{2}\right) \min \|\theta\|_{1} \text { subject to } y=\$ \% \phi \theta
$$

Due to the optimal spare solution $\theta^{*}$ of the problem $\left(\mathrm{P}_{2}\right)$, we can get the corresponding reconstructed multisignal ensembles $x^{*}=\psi^{*}$.

\section{Mobile-Agent-Based Adaptive Data Fusion Algorithm}

The DCS theory proposes a framework for joint reconstruction of compressible multi-signal ensembles. However, each single node independently encodes in DCS, which does not sufficiently utilize the joint sparsity of multi-signal ensembles. This operation makes each node have to transmit a lot of measurements. In this session, we focus on reducing measurements required to transmit at each node by mobile-agent-based data fusion in WSN.

A WSN under a DCS framework, as shown in Figure 1, consists of three types of components: Sink, sensor nodes and communication network. With energy restriction, sensor nodes can not directly communicate with Sink. For example, the encoding results of a node $S_{l+1}$ in the interesting field are transmitted to Sink by multi skips routing in Figure 1. Other nodes do the same works. In Figure 1 , we model a WSN as a graph $G=(V, E)$, where

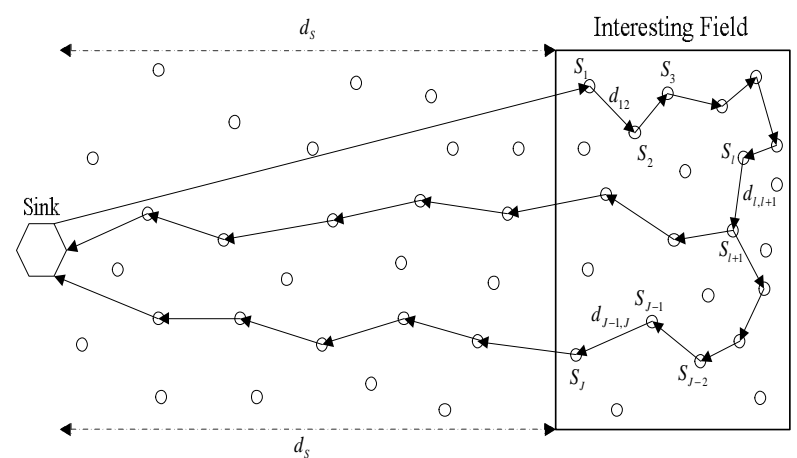

Figure 1. A WSN under DCS framework. Circles denote sensor nodes. The communication distance between node $S_{l}$ and node $S_{l+1}(l \in\{1, \mathrm{~L}, J-1\})$ is $d_{l, l+1}$ and the communication distance between Sink and $S_{l}$ is approximately as $d_{S}$ by the shortest multiple skips routing. For being simple, other links representing the communication links among nodes and Sink are omitted. On the other hand, a route $\left\{S_{S}, S_{1}, \mathrm{~L}, S_{J}, \mathrm{~L}, S_{S}\right\}$ represents MA data fusion routing. 
$V=\left\{S_{S}, S_{1}, \mathrm{~L}, S_{L}\right\}$ and $E$ denotes an edge set representing the communication links between node-pairs or links between Sink and nodes. In many applications, a distant Sink $S_{S}$ retrieves relevant interesting field information from the sensor nodes. Let $d_{S l}(l \in\{1, \mathrm{~L}, L\})$ be the total communication distance between Sink and a node $S_{l}(l \in\{1, \mathrm{~L}, L\})$ by the shortest multiple skips routing. For being simple, Sink is assumed to be far away from the interesting field so that $d_{S 1} \approx \mathrm{L} \approx d_{S L} \approx d_{S}$ and $d_{S} ? d_{l, l+1}(l \in\{1, \mathrm{~L}, L-1\})$, where $d_{l, l+1}$ denotes the communication distance between node-pairs $S_{l}$ and $S_{l+1}$ [13].

We next describe the communication architecture of WSN in Figure 1 to motivate the formulation of two data fusion algorithms for multiple signal ensembles. Assume that a connected subgraph $G^{\prime}=\left(V^{\prime}, E^{\prime}\right) \subseteq G$ is found, where $V^{\prime}$ contains Sink and encode nodes, i.e., $V^{\prime}=\left\{S_{S}, S_{1}, \mathrm{~L}, S_{J}\right\} \subset V \quad(I<H, J<L)$, and $E^{\prime}$ denotes a edge set representing all communication links in $V^{\prime}$. For a node $S_{l}(l \in\{1, \mathrm{~L}, J\})$, its node weight $w\left(S_{l}\right)$ denotes the amount of data outgoing from $S_{l}$. An edge $e_{l} \in E(l \in\{1, \mathrm{~L}, J-1\})$ is denoted by $e_{l}=\left(S_{l}, S_{l+1}\right)$. The weight of edge $e_{l}$ is equivalent to the weight of $S_{l}$, i.e., $w\left(e_{l}\right)=w\left(S_{l}\right)$. Two metrics, $t\left(e_{l}\right)$ and $f\left(e_{l}\right)$, are associated with each edge, describing the transmission cost and fusion cost on the edge, respectively. In many WSN applications, however, the fusion cost may be negligible. For being simple, we do not consider fusion cost in this paper.

The unit cost of the link for transmitting data from $S_{1}$ to $S_{l+1}$ is abstracted as $c\left(e_{l}\right)=\beta d_{l, l+1}^{r}+\varepsilon$, where $\beta$ and $r$ are tunable parameters based on the radio propagation [14]. Thus the transmission cost $t\left(e_{l}\right)$ is

$$
t\left(e_{l}\right)=c\left(e_{l}\right) w\left(e_{l}\right)
$$

Similarly, we approximately define $e_{S l}(l \in\{1, \mathrm{~L}, J\})$ is the communication links between a node $S_{l}$ and Sink by the shortest multiple skips routing, and then the transmission cost is approximately as $t\left(e_{S l}\right)=c\left(e_{S l}\right)$ $w\left(e_{S l}\right)(l \in\{1, \mathrm{~L}, J\})$, where $c\left(e_{S l}\right)$ and $w\left(e_{S l}\right)$ are the unit transmission cost and the weight of $e_{S l}$. Obviously, we can get $c\left(e_{S l}\right) ? c\left(e_{l}\right)$, for $d_{S} ? d_{l, l+1}$.

From above definitions, the total network energy consumption in $G^{\prime}=\left(V^{\prime}, E^{\prime}\right)$ with different computing models can be calculated. We firstly consider total network energy consumption of DCS. According to encoding method of a single signal, we assume that individual node $S_{l}(l \in\{1, \mathrm{~L}, J\})$ can gain a sparse coefficient vec- tor $\theta_{l}(l \in\{1, \mathrm{~L}, J\})$ by projecting a sensing signal $x_{l}(l \in\{1, \mathrm{~L}, J\})$ into a basis matrix $\Psi$. This operation may consume some computational energy, but it does not affect the total network energy consumption and can be omitted compared to transmission cost.

To conveniently explain the network energy consumption, we assume that $\Omega_{C} \cap \Omega_{l}=\varnothing$. Single signal reconstruction, therefore, needs $n_{l}$ measurements with $n_{l}=$ $c\left(k_{c}+k_{l}\right)$ in a CS framework, and then the total network energy consumption of DCS can be calculated as follows

$$
C_{D C S}=\sum_{l=1}^{J} c\left(e_{S l}\right) w\left(e_{S l}\right)=\sum_{l=1}^{J}\left(\beta d_{S}^{r}+\varepsilon\right) c\left(k_{C}+k_{l}\right)
$$

where the number of measurements $n_{l}=c\left(k_{C}+k_{l}\right)$ directly denotes the weight $w\left(e_{S l}\right)$.

We consider the network energy consumption of two mobile-agent-based data fusion algorithms. Generally speaking, MA is a special kind of software with small size, whose transmission cost can be omitted compared to transmission cost of larger amount of sensing information. We will not consider MA transmission cost. Sink predetermined the MA routing $\left\{S_{S}, S_{1}, \mathrm{~L}, S_{J}, \mathrm{~L}, S_{S}\right\}$ by GCF. The detailed mobile-agent-based data fusion process is presented as follows.

In initialize stage, MA migrates to $S_{1}$ and obtains measurements $y_{1}$ with $n_{1}=c\left\|\theta_{1}\right\|_{0}$. Subsequently, it migrates to $S_{2}$ and finds $\theta_{c} \hat{\theta_{1}}$ and $\hat{\theta_{2}}$ by contrasting sparse coefficients $\theta_{1}$ and $\theta_{2}$. Thus, it can calculate the common measurements $y_{c}$ corresponding to the common sparse component $\theta_{1}$ of the signal ensembles. This means that measurements $y_{1}$ and $y_{2}$ can be divided into $y_{1}=y_{C}+\hat{y}_{1}$ and $y_{2}=y_{C}+\hat{y}_{2}$, where $\hat{y}_{1}$ and $\hat{y}_{2}$ correspond to the sparse innovation component $\hat{\theta_{1}}$ and $\hat{\theta_{2}}$. MA carrying measurements $y_{C}, \hat{y}_{1}$ and $\hat{y}_{2}$ with $w\left(e_{2}\right)=c\left(k_{C}+\right.$ $k_{1}+k_{2}$ ) migrates to $S_{3}$. We then repeat above process on remaining nodes along MA routing until MA returns to Sink. Such process is a typical mobile-agent-based data fusion process. In this paper, we term this process a mobile-agent-based Full Data Fusion (FDF) algorithm compared to the following adaptive data fusion process. The total network energy consumption of FDF is shown as follows

$$
\begin{gathered}
C_{F D F}=\sum_{l=1}^{J-1} c\left(e_{l}\right) w\left(e_{l}\right)+c\left(e_{S J}\right) w\left(e_{S J}\right) \\
=\sum_{l=1}^{J-1}\left(\beta d_{l, l+1}^{r}+\varepsilon\right) c\left(k_{C}+k_{1}+\mathrm{L}+k_{l}\right) \\
\quad+\left(\beta d_{S}^{r}+\varepsilon\right) c\left(k_{C}+k_{1}+\mathrm{L}+k_{J}\right)
\end{gathered}
$$

We interest in comparing the total energy consumption 
of DCS and FDF. The total transmission cost can be calculated as follows

$$
\begin{gathered}
C_{D C S}-C_{F D F}=\left(\beta d_{S}^{r}+\varepsilon\right)(J-1) c k_{C}-\sum_{l=1}^{J-1}\left(\beta d_{l, l+1}^{r}+\varepsilon\right) c k_{l} \\
=\sum_{l=1}^{J-1}\left(\left(\beta d_{S}^{r}+\varepsilon\right) c k_{C}-\left(\beta d_{l, l+1}^{r}+\varepsilon\right) c k_{l}\right)
\end{gathered}
$$

Note that $d_{S} ? d_{l, l+1}$ and $k_{C}>k_{l}$, it is easy to get

$$
C_{D C S}>C_{F D F}
$$

As expected, the inequality (10) means that the total transmission cost of DCS is much larger than FDF. By analyzing FDF in detail, we find another challenge that MA will carry more and more amount of data fusion results along the MA routing. However, data fusion at a node $S_{l}(l \in\{2, \mathrm{~L}, J\})$ only needs the common measurements $y_{C}$. If MA still carries all front innovation measurements $\hat{y}_{1}, \mathrm{~L}, \hat{y}_{l-1}$, it is not in favor of saving communication cost. This result brings a question whether we can avoid transmitting an amount of the innovation measurements. In this regard, we establish the following data fusion algorithm named mobile-agentbased Adaptive Data Fusion (ADF) algorithm. Different from in FDF, MA only carries the common measurements $y_{C}$ in ADF. Concretely, MA can calculate $\hat{\theta_{l}}$ $(l \in\{2, \mathrm{~L}, J\})$ by contrasting $\theta_{C}$ and $\theta_{l}(l \in\{2, \mathrm{~L}$, $J\})$, when it carrying $y_{C}$ migrates to $S_{l}(l \in\{2, \mathrm{~L}, J\})$. This allows $y_{l}(l \in\{2, \mathrm{~L}, J\})$ to be divided into $y_{l}=y_{C}+\hat{y}_{l}$ in which the number of the innovation measurement $\hat{y}_{l}$ is $\hat{n}_{l}=c k_{l}$. MA carrying measurements $y_{C}$ with $w\left(e_{l}\right)=c k_{C}$ continuously migrates to $S_{l+1}$. On the other hand, measurements $\hat{y}_{l}$ are directly transmitted to Sink. We then repeat above process on remaining nodes along the same MA routing as FDF until MA returns to Sink. Then, the total network energy consumption of ADF is expressed as follows

$$
\begin{aligned}
& C_{A D F}=\left(\beta d_{12}^{r}+\varepsilon\right) c\left(k_{C}+k_{1}\right) \\
& +\sum_{l=2}^{J-1}\left(\left(\beta d_{l, l+1}^{r}+\varepsilon\right) c k_{C}+\left(\beta d_{S}^{r}+\varepsilon\right) c k_{l}\right)+\left(\beta d_{S}^{r}+\varepsilon\right) c\left(k_{C}+k_{J}\right)
\end{aligned}
$$

We also attend to compare the total energy consumption of FDF and ADF as follows

$$
\begin{gathered}
C_{F D F}-C_{A D F}=\sum_{l=1}^{J-1}\left(\beta d_{l, l+1}^{r}+\varepsilon\right) c k_{l}>0 \\
\text { i.e., } C_{F D F}>C_{A D F}
\end{gathered}
$$

From (10) and (13), it can be shown that

$$
C_{D C S}>C_{F D F}>C_{A D F}
$$

From (14), we can easily obtain the benefits of ADF.
Firstly, based on DCS, ADF also sufficiently utilizes both temporal (intra-signal) and spatial (inter-signal) correlations of multi-signal ensembles to analyze single signal sparsity structure and multi-signal ensembles jointly sparsity structure. These sparsity structures make it possible to perform data fusion of multi-signal ensembles. Second, the innovation measurements are allowed to directly transmit to Sink, while MA only carries the common measurements. It benefits reducing transmission cost. So we can say that ADF provides the optimal strategy for minimizing total transmission measurements and transmission cost compared to DCS and FDF.

According to the above discussion, we can reconstruct multi-signal ensembles by solving the following $l_{1}$ optimization problem

$$
\left(\mathrm{P}_{3}\right) \min \|\theta\|_{1} \text { subject to } \hat{y}=\hat{\Phi} \hat{\Psi} \theta
$$

$$
\begin{gathered}
\text { where } \theta=\left[\begin{array}{c}
\theta_{C} \\
\theta_{1} \\
\mathrm{M} \\
\theta_{J}
\end{array}\right], \hat{y}=\left[\begin{array}{c}
y_{C} \\
\hat{y}_{1} \\
\mathrm{M} \\
\hat{y}_{J}
\end{array}\right], \hat{\Phi}=\left[\begin{array}{cccc}
\Phi_{C} & 0 & \mathrm{~L} & 0 \\
0 & \Phi_{1} & \mathrm{~L} & 0 \\
\mathrm{M} & \mathrm{L} & \mathrm{O} & \mathrm{M} \\
0 & 0 & \mathrm{~L} & \Phi_{J}
\end{array}\right], \\
\hat{\Psi}=\left[\begin{array}{lll}
\Psi & \mathrm{L} & 0 \\
\mathrm{M} & \mathrm{O} & \mathrm{M} \\
0 & \mathrm{~L} & \Psi
\end{array}\right] .
\end{gathered}
$$

We can obtain $x^{*}=\left(x_{C}^{*}, \hat{x}_{1}^{*}, \mathrm{~L}, \hat{x}_{J}^{*}\right)^{T}$ with an optimal sparse solution $\theta^{*}=\left(\theta_{C}^{*}, \theta_{1}^{*}, \mathrm{~L}, \theta_{J}^{*}\right)^{T}$, where $x_{C}^{*}=\Psi \theta_{C}^{*}$ and $\hat{x}_{l}^{*}=\Psi \hat{\theta}_{l}^{*},(l \in\{1, \mathrm{~L}, J\})$. Furthermore, multi-signal ensembles can be reconstructed by $x_{l}^{*}=x_{C}^{*}+\hat{x}_{l}^{*}$, $(l \in\{1, \mathrm{~L}, J\})$.

The above results focus on theoretical analyses of saving transmission cost in ADF. On the other hand, we are interested in comparing the joint reconstruction performance of DCS, FDF and ADF. The following simulation results are presented illustrating the better joint reconstruction performance of ADF.

\section{Simulation}

In our setup, sensor nodes are randomly distributed in a region of a $50 \mathrm{~m} \times 50 \mathrm{~m}$ square. The distance between Sink and the interesting field is $d_{S}=400 \mathrm{~m}$. When considering transmission cost, we set $\beta=100 \mathrm{pJ} / \mathrm{bit} / \mathrm{m}^{2}$, $r=2$ and $\varepsilon=100 \mathrm{~nJ} /$ bit [12] in (6). Furthermore, we consider a series of example multiple signal ensembles $x_{1}, \mathrm{~K}, x_{J}$ that satisfy the conditions of joint sparsity model. The signal components $x_{C}, \hat{x}_{1}, \mathrm{~K}, \hat{x}_{J}$ are assumed to be sparse in Discrete Cosine Transform (DCT) matrix $\Psi$ with sparse parameters $k_{C}, k_{1}, \mathrm{~K}, k_{J}$, re- 


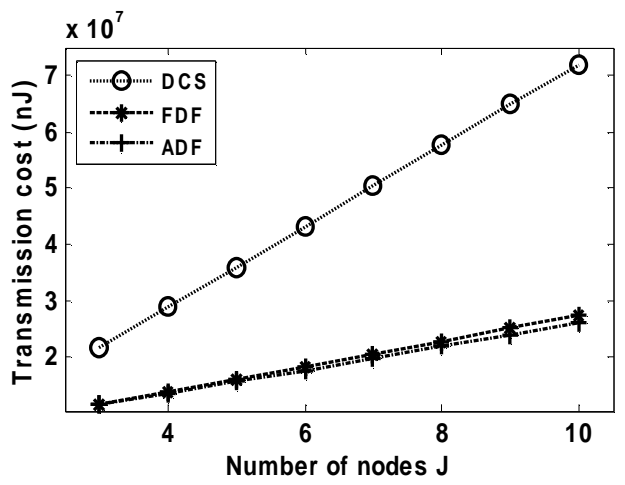

(a) The total transmission cost

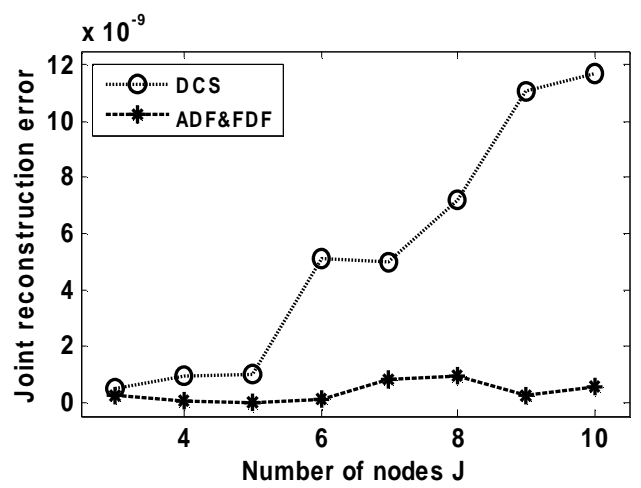

(c) Joint reconstruction error

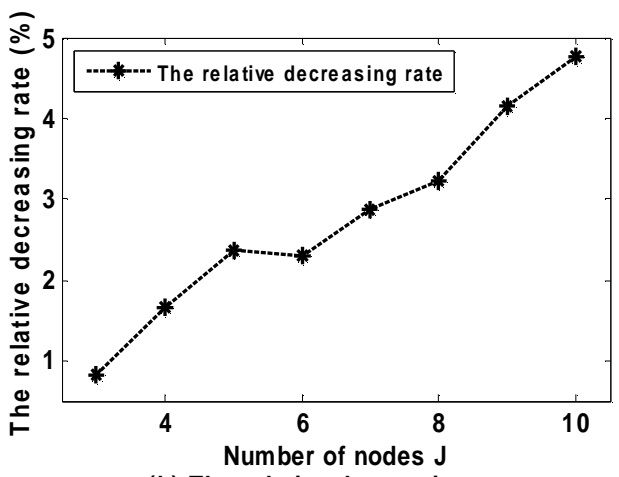

(b) The relative decreasing rate

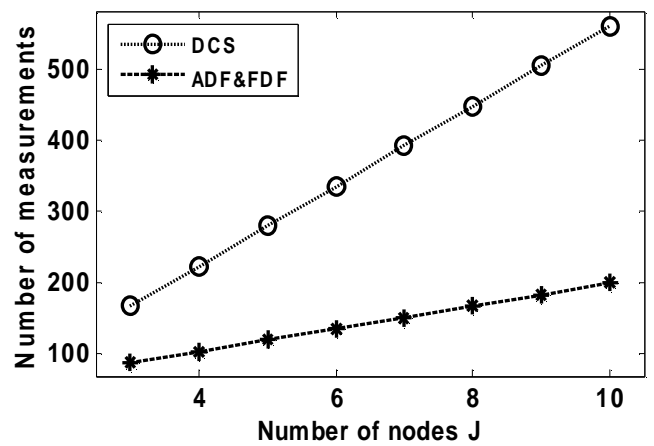

(d) The total number of measurements

Figure 2. Effect of the number of nodes on joint reconstruction performance of multiple signal ensembles. We choose signals with $m=50, k_{C}=10, k_{l}=4(l \in\{1, \mathrm{~L}, J\})$ and $n_{C}=4 k_{C}=40, \hat{n}_{l}=4 k_{l}=16(l \in\{1, \mathrm{~L}, J\})$. (a) The total network transmission cost, (b) The relative decreasing rate, (c) Joint reconstruction error, (d) The total number of measurements.

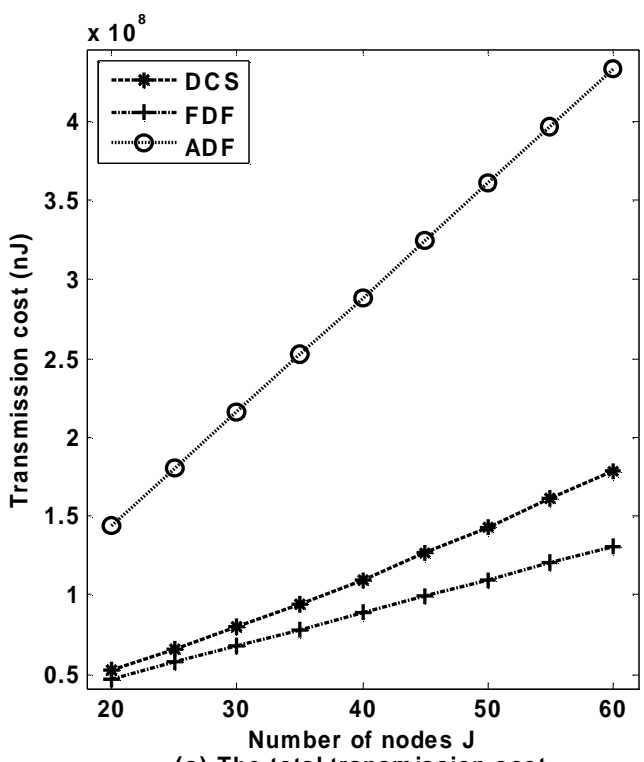

(a) The total transmission cost

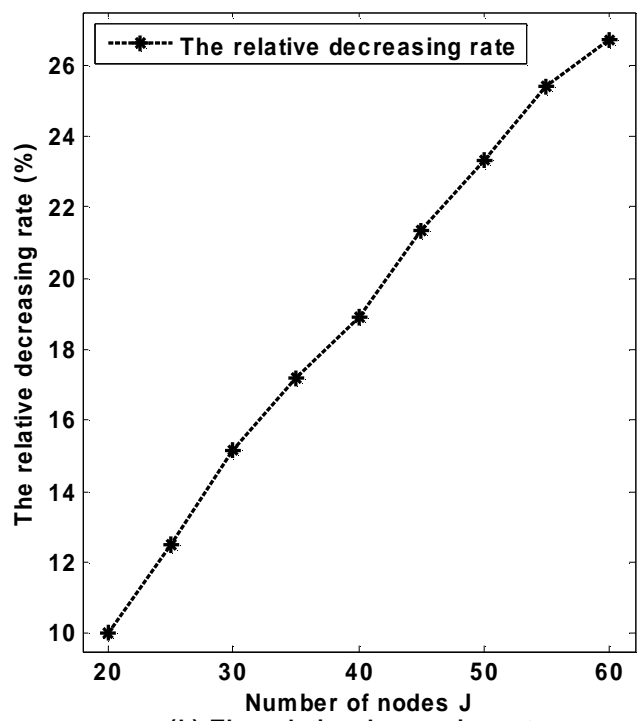

(b) The relative decreasing rate

Figure 3. Effect of the number of nodes on energy consumption of multiple signal ensembles. We choose signals with $m=50, k_{C}=10, k_{l}=4(l \in\{1, \mathrm{~L}, J\})$ and $n_{C}=4 k_{C}, \quad \hat{n}_{l}=4 k_{l}(l \in\{1, \mathrm{~L}, J\})$. (a) The total transmission cost, (b) The relative decreasing rate. 
spectively. We assign random Gaussian values to the nonzero coefficients $\theta_{C}, \hat{\theta_{1}}, \mathrm{~K}, \hat{\theta_{J}}$, and the locations of nonzero are chosen at random. As a measure of the reconstruction performance, the joint reconstruction error $e=\sum_{l=1}^{J}\left\|x_{l}-x_{l}^{*}\right\|_{2}$ is designed. The Interior Point

method in "Matlab" is used to solve the problem $\left(\mathrm{P}_{2}\right)$ and the problem $\left(\mathrm{P}_{3}\right)$.

Our first experiment chooses signals with the length $m=50$ and sparse parameters $k_{C}=10, k_{l}=4(l \in\{1, \mathrm{~L}, J\})$. Then the corresponding numbers of measurements are chosen by $n_{C}=c k_{C}=40, \hat{n}_{l}=c k_{l}=16(l \in\{1, \mathrm{~L}, J\})$, where $c=4$. Without loss of generality, assume that one measurement produces 8 bit packet [12]. With increasing number of nodes $J$, the total number of measurements of DCS is greatly larger than FDF and ADF in Figure 2(d). This result causes the transmission cost of DCS also greatly larger than FDF and ADF in Figure 2(a). To further illustrate the advantage of ADF, we consider the relative decreasing rate calculated as $\tau=\frac{\mathrm{TC}(\mathrm{FDF})-\mathrm{TC}(\mathrm{ADF})}{\mathrm{TC}(\mathrm{FDF})}$ in which $\mathrm{TC}(\mathrm{FDF})$ and $\mathrm{TC}(\mathrm{ADF})$ are the total transmission cost of FDF and ADF, respectively. Figure 2(b) clearly shows that the relative decreasing rate linearly increases with $J$. This means that the energy efficiency of $\mathrm{ADF}$ is more distinctness with increasing number of nodes. In Figure 2(c), we emphasize on comparison of reconstruction performance between DCS and ADF (FDF). ADF and FDF enjoy less joint reconstruction errors than DCS, though ADF and FDF use less number of measurements than DCS. So we can say that ADF performs much better than DCS and FDF.

WSN typically consists of a large number of sensor nodes, so we need consider energy consumption of much more nodes in DCS, FDF and ADF to further observe the advantage of ADF. We repeat the first experiment while the number of nodes varies from 20 to 60 . As expected, the transmission cost of DCS is further larger than FDF and ADF as $J$ increases in Figure 3(a). Comparing Figure 3(b) with Figure 2(b), we note that the relative decreasing rate scales linearly with $J$. The energy savings of ADF can be as large as $27 \%$. These results identify that $\mathrm{ADF}$ is an optimal strategy with minimum total number of measurements and total transmission cost, which consist with the front theoretical conclusions in Section 3.

Experiments in Figure 3 bring another question whether we can guarantee better joint reconstruction performance as the number of nodes $J$ increases. In our joint decoding simulations, we find that computational time and complexity will greatly increase as $J$ increases. So measurements in Sink should be grouped according to applications. This operation consists with the idea in [9]. In the next experiment, we use $J=40$ nodes and their measurements are separated in 5 groups. Average reconstruction error $\bar{e}=\left(\sum_{t=1}^{5} e_{t}\right) / 5$ is designed to measure the

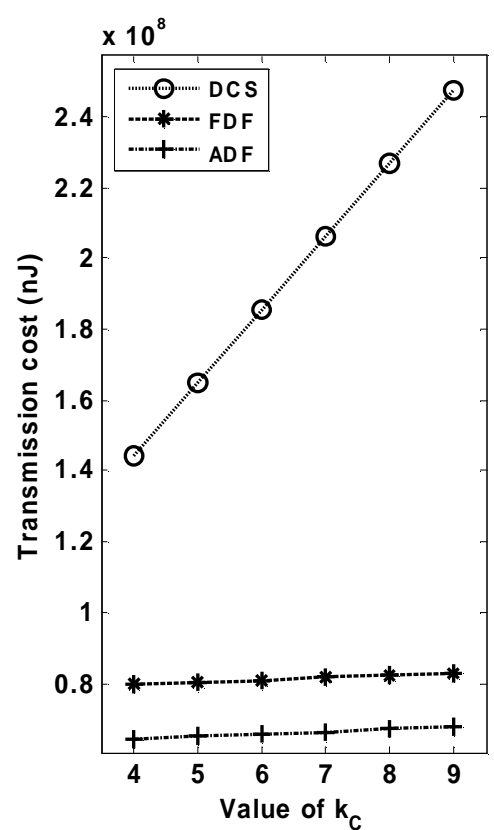

(a) The total transmission cost

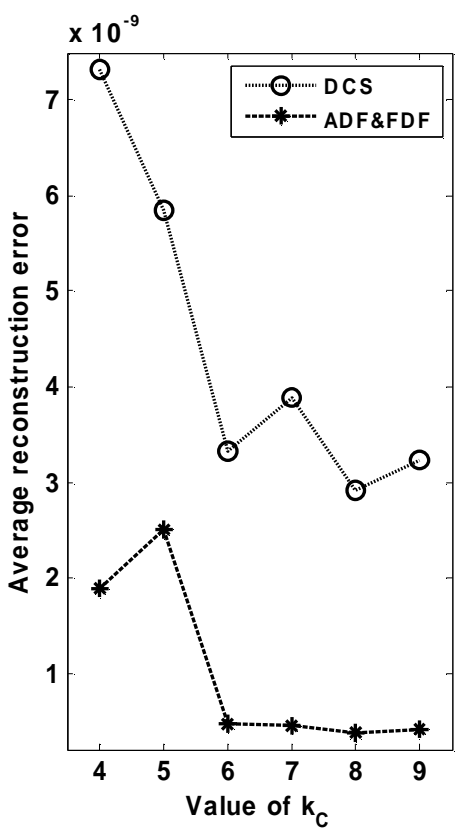

(b) Average reconstruction error

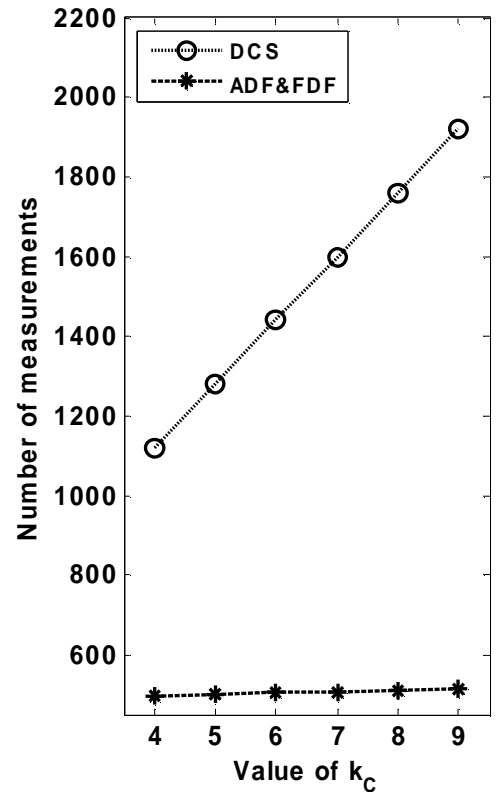

(c) The total number of measurements

Figure 4. Effect of the number of common sparse parameters $\boldsymbol{k}_{\boldsymbol{c}}$ on joint reconstruction of multiple signal ensembles. We choose $m=50$, fix $k_{l}=3(l \in\{1, \mathrm{~L}, J\})$, and vary common $\boldsymbol{k}_{\boldsymbol{c}}$ from 4 to 9 and then choose $n_{C}=4 k_{C}, \hat{n}_{l}=4 k_{l}(l \in\{1, \mathrm{~L}, J\})$. (a) The total transmission cost, (b) Average reconstruction errors, (c) The total number of measurements. 


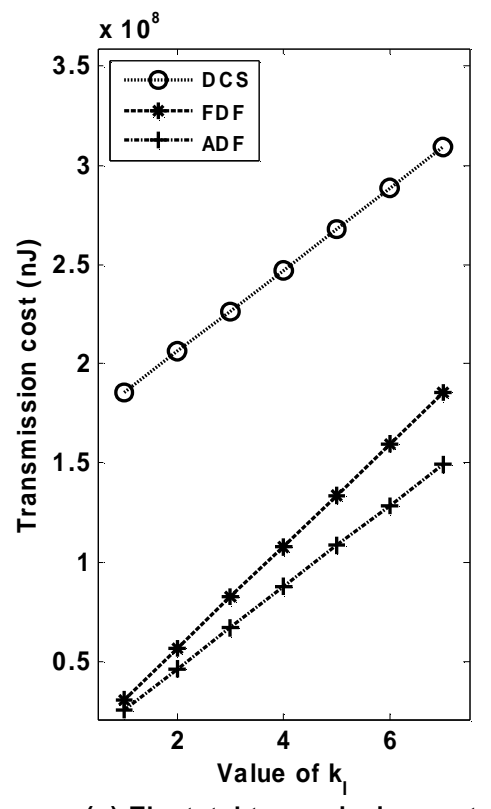

(a) The total transmission cost

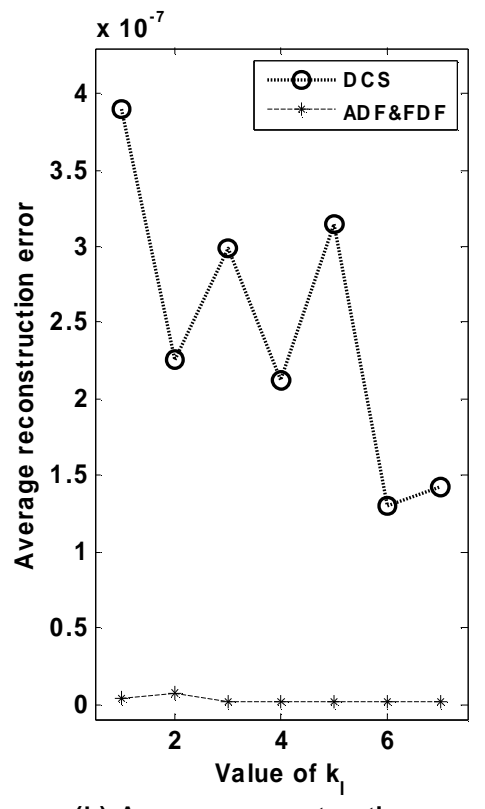

(b) Average reconstruction error

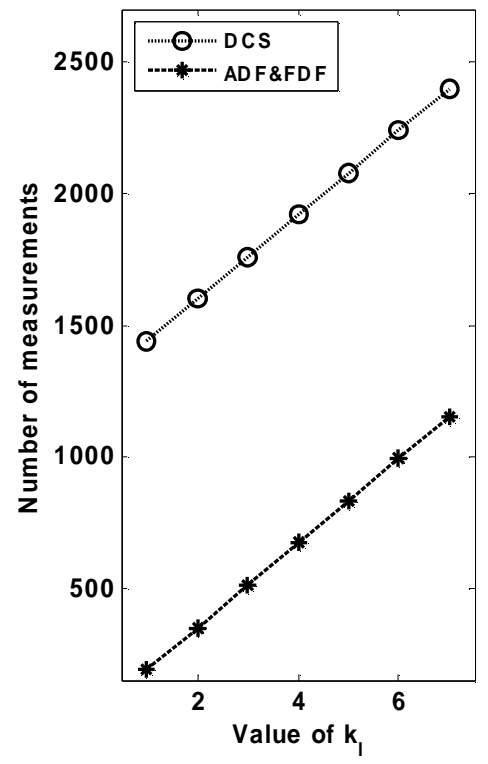

(c) The total Number of measurements

Figure 5. Effect of the number of innovation sparse parameters $k_{l}$ on joint reconstruction of multiple signal ensembles. We choose $m=50$, fix $k_{C}=8$ and $\operatorname{vary} k_{l}(l \in\{1, \mathrm{~L}, J\})$ from 1 to 7 , and then choose $n_{C}=4 k_{C}, \hat{n}_{l}=4 k_{l}(l \in\{1, \mathrm{~L}, J\})$. (a) The total transmission cost, (b) Average reconstruction errors, (c) The total number of measurements.

reconstruction performance, where $e_{t}(t=1, \mathrm{~L}, 5)$ is the joint reconstruction error of every group. We perform experiments with the length of signals $m=50$ and innovation sparse parameters $k_{l}=3(l \in\{1, \mathrm{~L}, J\})$, while the common sparse parameter $k_{c}$ varies from 4 to 9 . The obtained results in Figure 4 (a)-(c) show the similar conclusions in Figure 2 and Figure 3. The number of common sparse parameters $k_{c}$ greatly affects the total transmission cost of DCS while not FDF and ADF, for DCS need each node to transmit the common measurements while FDF and ADF avoid this operation by data fusion. This sufficiently reveals the advantage of mobile-agentbased data fusion algorithms. Moreover, the average reconstruction error of DCS, FDF and ADF decrease benefiting from the increasing number of measurements. This means joint reconstruction performance can be improved by increasing the total number of mea- surements.

The finally experiments focus on effect of the number of innovation sparse parameters $k_{l}$ on joint reconstruction of multiple signal ensembles. We repeat the front experiments with the length of signals $m=50$ and the common sparse parameter $k_{c}=8$, while the innovation sparse parameter $k_{l}(l \in\{1, \cdots J\})$ varies from 1 to 7 . As $k_{l}(l \in$ $\{1, \cdots J\})$ increasing, the total transmission cost and the number of measurements of FDF and ADF fleetly increase compared to Figure 4. Figure 5(a) and Figure 5(c) reveal that the performance of data fusion is influenced by the innovation sparse parameters $k_{l}$, for $k_{l}$ represent the differences among multi-signal ensembles. At the expense of more measurements and energy cost, we can obtain multi-signal ensembles with more details. At the same time, we gain the better joint reconstruction performance in Figure 5(b), for the total number of measurements increases.

As can be seen, above experiments imply that ADF sufficiently takes advantage of intra- and inter-signal correlation of multi-signal ensembles by mobile-agentbased data fusion. So ADF enjoys better performances than DCS and FDF.

\section{Conclusions}

Distributed Compressed Sensing (DCS) extends the theory and practice of Compressed Sensing (CS) to multisignal ensembles. A joint sparsity model for multi-signal ensembles with both intra- and inter-signal correlation captures the essence of real physical scenarios. This paper provides a new mobile-agent-based Adaptive Data Fusion (ADF) algorithm. ADF can greatly reduce the total number of measurements for successful joint reconstruction compared with DCS. Moreover, ADF can greatly reduce transmission cost and network load. Extensive experiments demonstrate that it indeed leads to 
better performance than DCS and mobile-agent-based Full Data Fusion (FDF).

\section{References}

[1] D. Donoho, "Compressed sensing," IEEE Trans. on Information Theory, Vol. 52, No. 4, pp. 1289-1306, April 2006.

[2] Y. Tsaig and D. Donoho, "Extensions of compressed sensing," Signal Processing, Vol. 86, No. 3, pp. 533-548, March 2006.

[3] J. Tropp and A. Gilbert, "Signal recovery from random measurements via orthogonal matching pursuit," IEEE Transactions on Information Theory, Vol. 53, No. 12, pp. 4655-4666, December 2007.

[4] Y. C. Kim, S. S. Narayanan, and K. S. Nayak, “Accelerated three-dimensional upper airway MRI using compressed sensing," Magnetic Resonance in Medicine, Vol. 61, pp. 1434-1440, 2009.

[5] M. Mishali and Y. C. Eldar, "Blind multi-band signal reconstruction: compressed sensing for analog signals," IEEE Transactions on Signal Processing, Vol. 57, No. 30, pp. 993-1009, March 2009.

[6] M. F. Duarte, S. Sarvotham, D. Baron, and M. B. Wakin, "Distributed compressed sensing of jointly sparse signals," in 39th Asilomar Conference on Signals, Systems and Computers, pp. 1537-1541, 2005.

[7] S. Pradhan and K. Ramchandran, "Distributed source coding using syndromes (DISCUS): Design and con- struction," IEEE Trans. on Information Theory, Vol. 49, pp. 626-643, 2003.

[8] Z. Xiong, A. Liveris, and S. Cheng, "Distributed source coding for sensor networks," IEEE Signal Processing Magazine, Vol. 21, pp. 80-94, September 2004.

[9] D. Baron, M. B. Wakin, M. F. Duarte, S. Sarvotham, and R. G. Baraniuk, "Distributed compressed sensing," http:// www.dsp.ece.rice.edu/cs.

[10] J. Meng, H. Li, and Z. Han, "Sparse event detection in wireless sensor networks using compressive sensing," The 43rd Annual Conference on Information Sciences and Systems (CISS'09), Baltimore, MD, 2009.

[11] A. H. Phan, A. Cichocki, and K. S. Nguyen, "Simple and efficient algorithm for distributed compressed sensing," IEEE Machine Learning for Signal Processing (MLSP' 08), pp. 61-66, October 2008.

[12] Q. Wu, N. S. V Rao, J. Barhen, S. S. Iyengar, V. K. Vaishnavi, H. Qi, and K. Chakrabarty, "On computing mobile agent routes for data fusion in distributed sensor networks, " IEEE Transactions on Knowledge and Data Engineering, Vol. 16, No. 6, pp. 740-753, June 2004.

[13] W. Bajwa, J. Haupt, and A. Sayeed, "Compressive wireless sensing," IPSN'06, Nashville, Tennessee, USA, pp. 19-21, 2006.

[14] H. Luo, J. Luo, Y. Liu, and S. K. Das, "Adaptive data fusion for energy efficient routing in wireless sensor networks," IEEE Transactions on Computers, Vol. 55, No. 10, pp. 1286-1299, October 2006. 